Marquette University

e-Publications@Marquette

Electrical and Computer Engineering Faculty

Research and Publications

Engineering, College of

$1-1-2017$

Axially Uniform Magnetic Field-Modulation Excitation for Electron Paramagnetic Resonance in Rectangular and Cylindrical Cavities by Slot Cutting

Jason Walter Sidabras

Medical College of Wisconsin

James Richie

Marquette University, james.richie@marquette.edu

James S. Hyde

Medical College of Wisconsin

Accepted version. Journal of Magnetic Resonance, Vol. 274 (January 2017): 115-124. DOI. (C) 2016

Elsevier Inc. Used with permission. 


\title{
Axially Uniform Magnetic Field- Modulation Excitation for Electron Paramagnetic Resonance in Rectangular and Cylindrical Cavities by Slot Cutting
}

\author{
Jason W. Sidebras \\ Department of Biophysics, Medical College of Wisconsin, \\ Milwaukee, WI \\ James E. Richie \\ Department of Electrical and Computer Engineering, \\ Marquette University, \\ Milwaukee, WI \\ James S. Hyde \\ Department of Biophysics, Medical College of Wisconsin, \\ Milwaukee, WI
}




\begin{abstract}
In continuous-wave (CW) Electron Paramagnetic Resonance (EPR) a low-frequency time-harmonic magnetic field, called field modulation, is applied parallel to the static magnetic field and incident on the sample. Varying amplitude of the field modulation incident on the sample has consequences on spectral line-shape and line-height over the axis of the sample. Here we present a method of coupling magnetic field into the cavity using slots perpendicular to the sample axis where the slot depths are designed in such a way to produce an axially uniform magnetic field along the sample. Previous literature typically assumes a uniform cross-section and axial excitation due to the wavelength of the field modulation being much larger than the cavity. Through numerical analysis and insights obtained from the eigenfunction expansion of dyadic Green's functions, it is shown that evanescent standing-wave modes with complex cross-sections are formed within the cavity. From this analysis, a W-band $(94 \mathrm{GHz})$ cylindrical cavity is designed where modulation slots are optimized to present a uniform $100 \mathrm{kHz}$ field modulation over the length of the sample.
\end{abstract}

\title{
Graphical abstract
}
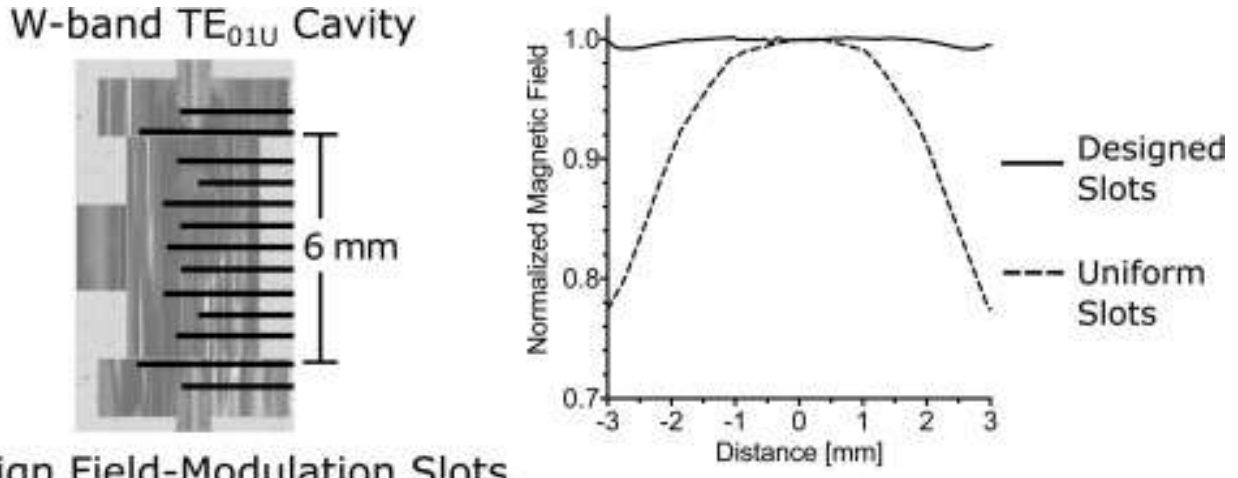

\section{Design Field-Modulation Slots}

Keywords: Waveguide evanescent modes, Dyadic Green's functions, Boundary conditions, Eigenvalues and eigenfunctions, Electromagnetic fields, Electron paramagnetic resonance

\section{Introduction}

The problem described here has applications in continuous-wave (CW) Electron Paramagnetic Resonance (EPR) where low-frequency time-harmonic magnetic field, typically $100 \mathrm{kHz}$, is applied parallel to a static magnetic field incident on a microwave cavity. The lowfrequency time-harmonic magnetic field, called field modulation, is coupled into the microwave cavity and modulates the resonance condition of the sample which offsets the EPR signal from the microwave carrier [1]. In order to maximize the field modulation incident on a sample the cavity can be designed in four ways: (i) the 
walls of the cavity are plated with silver that is electrically thin to the $100 \mathrm{kHz}$ field modulation but at least 10 microwave skin-depths thick, (ii) design the cavity as a wire-wound structure, (iii) place the fieldmodulation coil inside of the cavity, or (iv) cut slots transverse to the cavity microwave current to break up field-modulation eddy-currents on the outside of the cavity.

Previous work from our laboratory focused on field-modulation eddy-current analysis of silver-plated graphite resonators and the effect of the wall thickness with respect to the fields incident on the sample [2]. Here, we focus on how cutting transverse slots into the side walls couples the incident field modulation into the cavity and the cross-sectional field-modulation profile that is formed. This method is used at high frequency EPR (above $94 \mathrm{GHz}$ ) where methods ii and ii become problematic [3] and [4] or when modulation frequencies are increased for use in Electron Nuclear Double Resonance (ENDOR) EPR spectroscopy [5]. An illustration of the scheme studied in this work is shown in Fig. 1, where the modulation coils are a Helmholtz pair in the $y z$-plane. This configuration is known as a Helmholtz pair. Although this method focuses on field modulation it is applicable to ENDOR frequencies if the cavity and coil is rotated by 90 degrees around the $z$-axis.

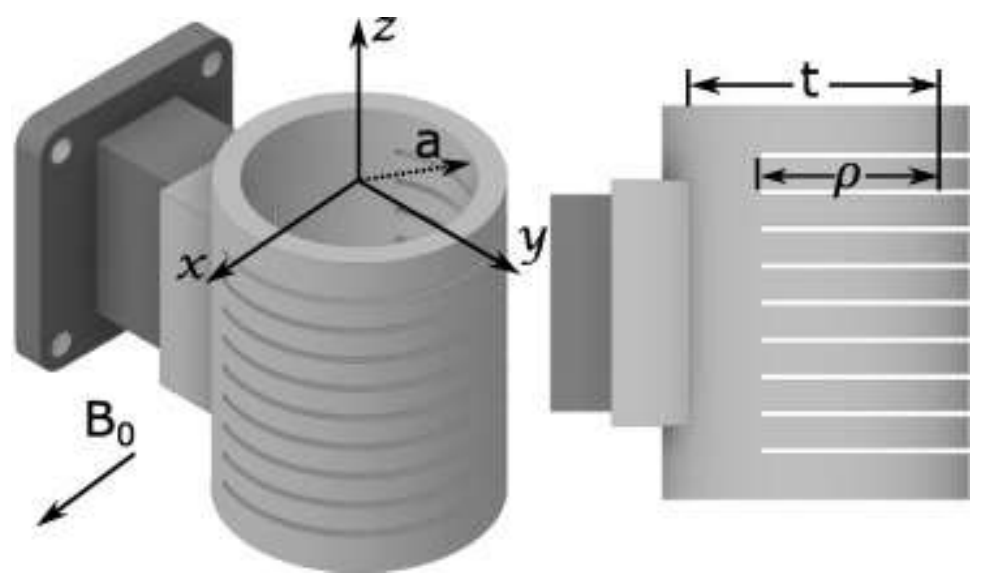

Fig. 1. A cylindrical $T E_{011}$ cavity resonator (without end-sections) is shown with waveguide coupling, field-modulation slots, and coordinate system. The static magnetic field and applied field modulation are in the $x$-direction. The inner diameter is $t$, with radius $a$, and the slot depth is $\rho$.

In general, the incident field modulation induces an electric field within the slots and evanescent modes are formed in the interior. To 
simplify the problem assume an infinitely long waveguide. EPR literature has not focused on the magnetic field cross-section of the induced field-modulation modes which has implications on sample lineshape, spin physics, and quantitative EPR [6]. Past and current literature assumes that the $100 \mathrm{kHz}$ field modulation has such a large wavelength compared to the resonator body that it can be regarded as quasi-static. This work shows that complex cross-section standingwaves modes form in the cavity and propagate as evanescent modes along the $z$ direction. These modes are formed from each slot and the interactions between slots. Understanding the coupling mechanism and field interactions gives insight into better EPR cavity design.

We present our results on two typical resonator geometries: a rectangular $\mathrm{TE}_{102}$ or cylindrical $\mathrm{TE}_{011}$ cavity. Here, the eigenfunction expansion for the dyadic Green's functions of magnetic types for rectangular and circular waveguides excited by a slot with an induced electric field induced by an externally homogeneous magnetic field are formulated. The eigenfunction expansion of $\overline{\bar{G}}_{m}$, known as the OhmRayleigh method, is explicitly used to derive the dyadic Green's function of the magnetic field within the waveguide geometries [7] ; [8]. The magnetic field solution for a single slot along the waveguide axis and a cross-sectional profile is presented. Multiple-slot geometry derivation is described using a simple summation (zeroorder) of the individual slots and a method of moments (first-order) modification for slot-to-slot interactions. Ansys High Frequency Structure Simulator (HFSS; v. 17.0, Canonsburg, PA) is utilized to both validate and normalize the Green's function solutions. Good agreement is shown between Green's function and numerical data. The combined first-order method of moments dyadic Green's function solution for two slots gives insight on the interactions of multiple slots and yields phenomenological guidance in the design of EPR cavities.

From the insight gained, we describe a cylindrical cavity at Wband with modulation slots designed with varying depths which present a uniform $100 \mathrm{kHz}$ field modulation on axis over the length of the sample. Additionally, the cylindrical cavity is a $T_{01 U}$, where the $U$ subscript denotes that the microwave magnetic field is also uniform in the $z$-direction over the sample. It has been shown that the microwave magnetic field in a cavity can be made uniform by designing the cavity

Journal of Magnetic Resonance, Vol 274 (January 2017): pg. 115-124. DOI. This article is @ Elsevier and permission has been granted for this version to appear in e-Publications@Marquette. Elsevier does not grant permission for this article to be further copied/distributed or hosted elsewhere without the express permission from Elsevier. 
as a waveguide section at cut-off over the region of interest and proper end-sections to tune the cavity to the cut-off frequency [9]; [10] ; [11]. This work presents the first uniform field-modulation and microwave magnetic field cavity resonator at $\mathrm{W}$-band.

\section{Methods}

The dyadic Green's functions are derived using a number of references cited in this work and are solved by programming them into Wolfram (Champaign-Urbana, IL) Mathematica (version 10.0). Mathematica includes pre-defined parallel programming functions such as ParallelSum and ParallelTable. Using these functions, solutions to the dyadic Green's functions were accelerated by $76 \%$ resulting in solution times of approximately 2 min.

Parameters such as the electric field amplitude and profile within the slot are taken from Ansys HFSS solutions and used in the formulation of the dyadic Green's functions. This ensures that the dyadic Green's functions and HFSS solutions are directly comparable. Once all solutions are formed, the Green's function and numerical solutions are compared using both visual and analytical techniques. Visually, a two dimensional cross-section solution of the solved waveguide is plotted in a side-by-side comparison to view contour similarities.

Since this work focuses on an axial $100 \mathrm{kHz}$ magnetic field, one must ensure the evanescent roll-off and field amplitude profile are properly reflected in the dyadic Green's function. A root-mean-square error (RMSE) function was employed to calculate the residual error between the normalized Green's function and numerical results. Using a RMSE is an accurate measurement to compare a calculated model (the dyadic Green's function) to the full-wave 3D simulation (Ansys HFSS) and has the unites of amps per meter $(A / m)$. In order to calculate the RMSE both equations are discretized into $n$ segments and directly compared according to 
RMSE $=\sqrt{\frac{1}{n} \sum_{m=1}^{n}\left(H_{\text {cal }}-H_{\text {sim }}\right)^{2}}[\mathrm{~A} / \mathrm{m}]$.

where $\mathrm{H}_{c a l}$ and $\mathrm{H}_{\text {sim }}$ are the magnetic field calculated by the dyadic Green's functions and the magnetic field simulated by Ansys HFSS, respectively. Both the visual and analytical analysis give confidence in the dyadic Green's function solutions to form a resulting insight and discussion of this work.

In order to minimize Mathematica calculation time, the number of modes that were solved in the analytical code was varied until the solutions had an acceptable convergence. It was found that using ten TE and nine TM evanescent modes resulted in more than adequate convergence. These results were consistent in rectangular and cylindrical waveguides.

\section{Eigenfunction expansion of dyadic Green's functions}

Time-harmonic electric and magnetic fields, $\mathrm{e}^{-\mathrm{i} \omega \mathrm{t}}$, are assumed throughout the formulation of the problem and solutions.

\subsection{Rectangular waveguide formulation}

The rectangular waveguide is defined in Fig. 2, where $\hat{\mathbf{z}}$ is the propagation vector and the electromagnetic modes are bounded by $0 \leqslant x \leqslant a$ and $0 \leqslant y \leqslant b$. The vector wave equation is defined as

\section{$\nabla \times \nabla \times \bar{\Phi}-\kappa^{2} \bar{\Phi}=0$,}

where $\mathrm{k}$ and $\bar{\Phi}$ satisfy the Helmholtz wave equation given the appropriate boundary functions. Since a rectilinear coordinate system is used, the Cartesian or rectilinear vector wave function is used: 


\section{$\bar{\Phi}=\mathbf{\nabla} \times\left(\psi_{1} \hat{\mathbf{e}}\right)$,}

equation(3)

where $\mathbf{e}$ denotes a constant vector and $\psi_{1}$ is the characteristic function that satisfies the scalar wave equation

$\nabla^{2} \psi_{1}+\kappa^{2} \psi_{1}=0$

equation(4)

The function $\psi_{1}$ is known as the generating function, and $\mathbf{\mathbf { e }}$ is known as the piloting vector. It can be shown that Eq. (3) is a solution of Eq. (2) if $\psi_{1}$ is a solution for Eq. (4). The set of functions obtained, denoted by $\bar{M}_{\bar{\varphi}}$, is

\section{$\bar{M}_{\mathscr{*}}=\boldsymbol{\nabla} \times\left(\psi_{1} \hat{\mathbf{e}}\right)$.}

equation(5)

Similarly, another set of functions, denoted by $\bar{N}_{\bar{\phi}}$, is described by

$\bar{N}_{\varphi}=\frac{1}{\kappa} \nabla \times \nabla \times\left(\psi_{2} \hat{\mathbf{e}}\right)$

equation(6)

where $\Psi_{2}$ does not have to equal $\Psi_{1}$, but both must satisfy Eq. (4).

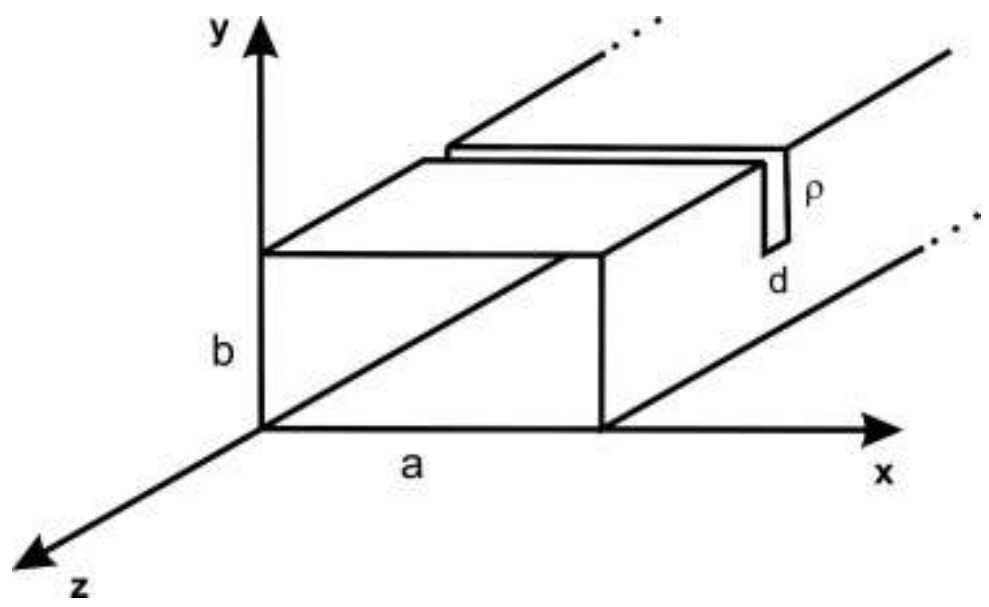

Journal of Magnetic Resonance, Vol 274 (January 2017): pg. 115-124. DOI. This article is (C) Elsevier and permission has been granted for this version to appear in e-Publications@Marquette. Elsevier does not grant permission for this article to be further copied/distributed or hosted elsewhere without the express permission from Elsevier. 
Fig. 2. Definition of the rectangular geometry with a slot thickness of $d$ and a depth of $\rho$ cut into the broad face of the waveguide. The walls of the waveguide are perfect electric conductor (PEC) material and have a cross-section of $a$ by $b$. The waveguide wall thickness is not finite, but propagation is assumed instantaneous.

Using the separation of variables method, the general solution to the scalar wave equation in rectangular coordinates can be written as

equation(7)

$\psi=\left[A \cos \left(k_{x} x\right)+B \sin \left(k_{y} y\right)\right] \times\left[C \cos \left(k_{y} y\right)+D \sin \left(k_{x} x\right)\right] e^{i h z}$,

where $k_{x}^{2}+k_{y}^{2}+h^{2}=\kappa^{2}$ and bounded by $0 \leqslant x \leqslant a, 0 \leqslant y \leqslant b$ and $-\infty<z<\infty$. Here, $h$ denotes the propagation constant of the wave in $\pm \hat{\mathbf{Z}}$ coordinate and $k_{x}$ and $k_{y}$ denote standing waves in $\hat{\mathbf{x}}$ and $\hat{\mathbf{y}}$, respectively.

The vector wave functions, both $\bar{M}_{\bar{\phi}}$ and $\bar{N}_{\bar{\phi}}$, satisfy the vector Dirichlet boundary conditions

\section{$\hat{\mathbf{n}} \times \bar{M}_{\varphi}=0$ and}

equation(8)

$\hat{\mathbf{n}} \times \bar{N}_{\mathscr{\Phi}}=0$

equation(9)

on the waveguide walls.

Since the propagation direction, or piloting vector, is in the $\hat{\mathbf{Z}}$ direction, $\hat{\mathbf{e}}$ is replaced by $\hat{\mathbf{z}}$. Then the complete $\bar{M}_{\phi}$ and $\bar{N}_{\phi}$ functions, which satisfy the vector Dirichlet condition, are

$$
\begin{aligned}
\bar{M}_{\text {emn }}(h) & =\nabla \times\left[\psi_{\text {emn }}(h) \hat{\mathbf{z}}\right] \\
& =\nabla \times\left[\cos \left(k_{x} x\right) \cos \left(k_{y} y\right) e^{i h z} \hat{\mathbf{z}}\right] \text { and }
\end{aligned}
$$




$$
\begin{aligned}
\bar{N}_{\text {omn }}(h) & =\frac{1}{\kappa} \boldsymbol{\nabla} \times \boldsymbol{\nabla} \times\left[\psi_{\text {emn }}(h) \hat{\mathbf{z}}\right] \\
& =\frac{1}{\kappa} \boldsymbol{\nabla} \times \boldsymbol{\nabla} \times\left[\sin \left(k_{x} x\right) \sin \left(k_{y} y\right) e^{i h \mathbf{z}} \hat{\mathbf{z}}\right] .
\end{aligned}
$$

The subscript "o" is used to denote "odd," and the subscript "e", "even." It is understood that odd functions with $m$ or $n$ equal to zero are null modes. The vector functions that properly represent the magnetic field in a rectangular waveguide can be found by

equation(12)

$$
\begin{aligned}
\bar{M}_{\text {omn }}(h)= & \frac{1}{\kappa} \nabla \times \bar{N}_{\text {omn }} \\
= & \left(k_{y} \sin \left(k_{x} x\right) \cos \left(k_{y} y\right) \hat{\mathbf{x}}\right. \\
& \left.-k_{x} \cos \left(k_{x} x\right) \sin \left(k_{y} y\right) \hat{\mathbf{y}}\right) e^{\text {ihz }} \text { and }
\end{aligned}
$$

$$
\begin{aligned}
\bar{N}_{\text {emn }}(h)= & \frac{1}{\kappa} \nabla \times \bar{M}_{\text {emn }} \\
= & \frac{1}{\kappa}\left(i h k_{x} \sin \left(k_{x} x\right) \cos \left(k_{y} y\right) \hat{\mathbf{x}}\right. \\
& -i h k_{y} \cos \left(k_{x} x\right) \sin \left(k_{y} y\right) \hat{\mathbf{y}} \\
& \left.+k_{c}^{2} \cos \left(k_{x} x\right) \cos \left(k_{y} y\right) \hat{\mathbf{z}}\right) e^{i h z} .
\end{aligned}
$$

The functions $\bar{M}_{\text {omn }}(h)$ and $\bar{N}_{\text {emn }}(h)$ satisfy the vector Neumann condition on the boundary, mainly,

$\hat{\mathbf{n}} \times \nabla \times \bar{M}=0$ and

equation(14)

$\hat{\mathbf{n}} \times \nabla \times \bar{N}=0$.

equation(15) 
For this problem, we use the Neumann boundary conditions for formulating the magnetic field solutions.

Using the Ohm-Rayleigh method, the normalization factor is determined for the functions

$$
\begin{gathered}
\int_{0}^{a} \int_{0}^{b} \int_{-\infty}^{\infty} \bar{M}_{o m n}(h) \cdot \bar{M}_{o m n}\left(-h^{\prime}\right) d x d y d z \\
\quad=\int_{-\infty}^{\infty}\left(1+\delta_{0}\right) \frac{\pi a b k_{c}^{2}}{2} \delta\left(h-h^{\prime}\right) d z
\end{gathered}
$$

where the $\delta_{0}$ function denotes the Kronecker delta function, which is 1 if $\mathrm{m}=\mathrm{n}$ and 0 otherwise. The null modes of $\bar{M}_{\mathrm{mmn}}(h)$ are included in this normalization since when $m$ or $n$ is zero, $\bar{M}_{\text {omn }}(h)$ has a normalization of zero. Similarly, the normalization of the $\bar{N}_{\text {emun }}$ function can be found by using

$$
\begin{gathered}
\int_{0}^{a} \int_{0}^{b} \int_{-\infty}^{\infty} \bar{N}_{\text {emn }}(h) \cdot \bar{N}_{\text {emn }}\left(-h^{\prime}\right) d x d y d z \\
=\int_{-\infty}^{\infty}\left(1+\delta_{0}\right) \frac{\pi a b k_{c}^{2}}{2} \delta\left(h-h^{\prime}\right) d z .
\end{gathered}
$$

equation(17)

Once the vector wave functions are properly defined, the dyadic Green's function can be constructed. The eigenfunctions defined in $\bar{M}_{\Phi}$ and $\bar{N}_{\bar{\phi}}$ allow for the complete vector solution for the magnetic field in the waveguide.

\subsection{The method of $\overline{\bar{G}}_{m}$}

The method of $\overline{\bar{G}}_{m}$ uses the Ohm-Rayleigh method to derive the magnetic dyadic Green's function of the second kind (Neumann boundary) for a rectangular waveguide using the vector wave equations [7]; [8]; [12] ; [13]. The dyadic Green's function, denoted $\overline{\bar{G}}_{m 2}\left(\bar{r} ; \bar{r}^{\prime}\right)$, must satisfy the equation 
$\nabla \times \nabla \times \overline{\bar{G}}_{m 2}\left(\bar{r}_{;} \bar{r}^{\prime}\right)-k^{2} \overline{\bar{G}}_{m 2}\left(\bar{r}_{;}, \bar{r}^{\prime}\right)=\nabla \times\left[\overline{\bar{I}} \delta\left(\bar{r}-\bar{r}^{\prime}\right)\right]$ and

equation(19)

\section{$\hat{\mathbf{n}} \times \boldsymbol{\nabla} \times \overline{\bar{G}}_{m 2}\left(\bar{r}_{\xi}^{*} \bar{r}^{\prime}\right)=0$}

Using the Ohm-Rayleigh method, an eigenvalue expansion is derived for the source function $\nabla \times\left[\overline{\bar{I}} \delta\left(\bar{r}-\bar{r}^{\prime}\right)\right]$ using the functions introduced earlier. The functions $\bar{N}_{\text {emn }}(h)$ and $\bar{M}_{\text {omn }}(h)$ are used for the magnetic field solutions and to satisfy the Neumann boundary conditions. Expanding the source function using the vector wave functions yields

$\nabla \times\left[\overline{\bar{I}} \delta\left(\bar{r}-\bar{r}^{\prime}\right)\right]=\int_{-\infty}^{\infty} d h \sum_{m=0}^{\infty} \sum_{n=0}^{\infty}\left[\bar{N}_{e m n}(h) \bar{A}_{e m n}(h)+\bar{M}_{o m n}(h) \bar{B}_{o m n}(h)\right]$

where it can be shown that

equation(21)

$\bar{A}_{\text {emn }}(h)=\frac{\left(2-\delta_{0}\right) \kappa}{\pi a b k_{c}^{2}}\left(-\hat{\mathbf{n}} \cdot\left[\bar{N}_{\text {emn }}(h) \times \overline{\bar{I}} \delta\left(\bar{r}-\bar{r}^{\prime}\right)\right]\right)$ and

equation(22)

$\bar{B}_{o m n}(h)=\frac{\left(2-\delta_{0}\right) \kappa}{\pi a b k_{c}^{2}}\left(-\hat{\mathbf{n}} \cdot\left[\bar{M}_{o m n}(h) \times \overline{\bar{I}} \delta\left(\bar{r}-\bar{r}^{\prime}\right)\right]\right)$.

Since the rectangular waveguide extends to infinity in $\hat{\mathbf{Z}}$ and since the integrand has two poles at $h=+/-\left(k^{2}-k_{c}^{2}\right)^{\frac{1}{2}}$, because $k$ is complex, the solution meets the requirements of the Jordan lemma in the theory of complex variables [14]. Finally, the Green's function for the magnetic field satisfying the boundary condition of the second type can be written as 


$$
\begin{aligned}
\overline{\bar{G}}_{m 2}\left(\bar{r}_{;} \bar{r}^{\prime}\right)= & \frac{i k}{a b} \sum_{m=0 n=0}^{\infty} \sum_{0}^{\infty} \frac{\left(2-\delta_{0}\right)}{k_{c}^{2} k_{g}}\left[\bar{N}_{e m n}\left( \pm k_{g}\right) \bar{A}_{\text {emn }}^{\prime}\left(\mp k_{g}\right)\right. \\
& \left.+\bar{M}_{o m n}\left( \pm k_{g}\right) \bar{B}_{o m n}^{\prime}\left(\mp k_{g}\right)\right],
\end{aligned}
$$

equation(23)

where $z>z^{\prime}$ corresponds to \pm and $z<z^{\prime}$ corresponds to $\mp$ for $k_{g}$ and

$$
\begin{aligned}
& \bar{A}_{\text {emn }}^{\prime}\left(k_{g}\right)=-\hat{\mathbf{n}} \cdot\left[\bar{N}_{\mathrm{em} n}^{\prime}\left(\mp k_{g}\right) \times \overline{\bar{I}} \delta\left(\bar{r}-\bar{r}^{\prime}\right)\right], \text { and } \\
& \bar{B}_{\text {omn }}^{\prime}\left(k_{g}\right)=-\hat{\mathbf{n}} \cdot\left[\bar{M}_{\text {omm }}^{\prime}\left(\mp k_{g}\right) \times \overline{\bar{I}} \delta\left(\bar{r}-\bar{r}^{\prime}\right)\right] .
\end{aligned}
$$

By integrating over the closed surface of the magnetic current source, $\bar{M}_{s}$, on the surface of the waveguide walls such that

equation(26)

$$
\bar{H}(x, y, z)=i \omega \int_{S} \overline{\bar{G}}_{m 2}\left(x, y, z_{;} x^{\prime}, y^{\prime}, z^{\prime}\right) \cdot \bar{M}_{s}\left(x^{\prime}, y^{\prime}, z^{\prime}\right) d S,
$$

equation(25)

the solution to the magnetic field using $\overline{\bar{G}}_{m}$ is found. The volume integral of the Green's function vanishes because the excitation is on the waveguide walls.

The solution to Eq. (26) is valid everywhere within the domain except at the source surface. In this work, Eq. (26) is exclusively used to find the magnetic field in a rectangular waveguide. This derivation is necessary not only for full understanding of the solution, but also because previous derivations, in both Tai [7] ; [8] and Ho et al. [15], derive solutions for sources within the waveguide, not on the waveguide walls.

\subsection{Cylindrical waveguide formulation}

Formulation of the cylindrical waveguide, depicted in Fig. 3 follows the same steps as the rectangular waveguide [7] ; [8]. In this case the characteristic equation uses cylindrical Bessel functions as the eigenfunction expansions. Again, $\hat{\mathbf{z}}$ is the propagation vector and the

Journal of Magnetic Resonance, Vol 274 (January 2017): pg. 115-124. DOI. This article is (C) Elsevier and permission has been granted for this version to appear in e-Publications@Marquette. Elsevier does not grant permission for this article to be further copied/distributed or hosted elsewhere without the express permission from Elsevier. 
cylindrical modes are bounded by $0 \leqslant r \leqslant a$. The characteristic wave equation is then determined by

$\psi_{e_{n}}=J_{n}\left(\frac{\gamma_{m n}^{\prime}}{a} r\right)\left(\begin{array}{c}\cos (n \phi) \\ \sin \end{array}\right) e^{i h z}$, and

equation(27)

$\psi_{e_{n} n \gamma}=J_{n}\left(\frac{\gamma_{m n}}{a} r\right)\left({ }_{\sin }^{\cos }(n \phi)\right) e^{i h z}$,

equation(28)

where, $a$ is the radius of the cylindrical waveguide and $\gamma_{m n}$ and $\gamma_{m n}^{\prime}$ are the zeros associated with the Bessel function of the first kind or the derivative of the Bessel function of the first kind, respectively. The subscript 'e ' denotes an even function where $\cos (n \varphi)$ is used and the subscript 'o ' denotes an odd function where $\sin (n \varphi)$ is used. The wave constant for the two wave equations is defined as

$k_{\gamma}^{2}=\left(\frac{\gamma_{m} n}{a}\right)^{2}+h^{2}$, and

equation(29)

$k_{\gamma}^{2}=\left(\frac{\gamma_{m}^{\prime} n}{a}\right)^{2}+h^{2}$

equation(30)

Then the cylindrical vector wave functions can be defined by

$\bar{M}_{e^{m} m \gamma}(h)=\nabla \times \psi_{e_{a} m \gamma} \hat{\mathbf{z}}$, and

equation(31)

$\bar{N}_{e_{a} m \gamma}(h)=\frac{1}{k_{\gamma}} \nabla \times \nabla \times \psi_{e_{a} m \gamma} \hat{\mathbf{z}}$,

equation(32)

where both satisfy vector Dirichlet boundary conditions at $r=a$. Using the Ohm-Rayleigh method and the method of $\overline{\bar{G}}_{m}$ as described in the 
previous section the dyadic Green's function for the cylindrical waveguide can be expressed as

$$
\text { equation(33) }
$$

where $z>z^{\prime}$ corresponds to \pm and $z<z^{\prime}$ corresponds to $\mp$ for $k_{g}$ and

$$
\begin{aligned}
& \bar{A}_{a}^{\prime}{ }_{n \gamma}\left(k_{\gamma}\right)=-\hat{\mathbf{n}} \cdot\left[\bar{M}_{e_{n \gamma \gamma}}\left(\mp k_{\gamma}\right) \times \overline{\bar{I}} \delta\left(\bar{r}-\bar{r}^{\prime}\right)\right], \text { and } \\
& \bar{B}_{e_{n \gamma}}\left(k_{\gamma}\right)=-\hat{\mathbf{n}} \cdot\left[\bar{N}_{e_{n \gamma}}\left(\mp k_{\gamma}\right) \times \overline{\bar{I}} \delta\left(\bar{r}-\bar{r}^{\prime}\right)\right] .
\end{aligned}
$$

Additionally the constants $c_{Y}{ }^{\prime}$ and $c_{Y}$ are defined as

$$
\begin{aligned}
& c_{\gamma}=\frac{i\left(2-\delta_{0}\right)}{4 \pi\left(\frac{\gamma}{a}\right)^{2} k_{\gamma} I_{\gamma}}, \text { and } \\
& c_{\gamma}=\frac{i\left(2-\delta_{0}\right)}{4 \pi\left(\frac{\gamma}{a}\right)^{2} k_{\gamma} I_{\gamma}} .
\end{aligned}
$$

Finally, the scaling constants $\mathrm{I}_{Y}{ }^{\prime}$ and $\mathrm{I}_{\mathrm{Y}}$ are defined as

$$
\begin{aligned}
& I_{\gamma}=\frac{a^{2}}{2\left(\frac{\gamma}{a}\right)^{2}}\left(\left(\frac{\gamma}{a}\right)^{2}-\frac{n^{2}}{a^{2}}\right) J_{n}^{2}\left[\left(\frac{\gamma}{a}\right)^{2} a\right], \text { and } \\
& I_{\gamma}=\frac{a^{2}}{2\left(\frac{\gamma}{a}\right)^{2}}\left[\frac{\partial J_{n}\left(\frac{z}{a} r\right)}{\partial r}\right]_{r=a}^{2} .
\end{aligned}
$$



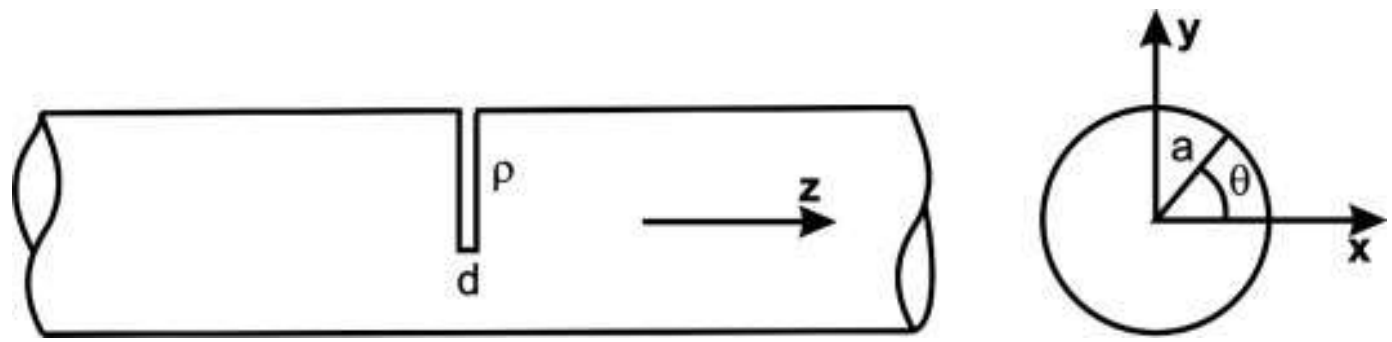

Fig. 3. Definition of the cylindrical geometry with a slot thickness of $d$ and a depth of $\rho$ cut into the broad face of the waveguide. The walls of the waveguide are PEC material and have a radius of $a$. The waveguide wall thickness is not finite, but propagation is assumed instantaneous.

Integrating the magnetic current source, $\bar{M}_{s}$, on the closed surface of the waveguide walls produces the solution,

$$
\bar{H}(r, \phi, z)=i \omega \int_{S} \overline{\bar{G}}_{m 2}\left(r, \phi, z_{z}^{*} r^{\prime}, \phi^{\prime}, z^{\prime}\right) \cdot \bar{M}_{s}\left(r^{\prime}, \phi^{\prime}, z^{\prime}\right) d S .
$$

\subsection{Single-slot formulation}

The incident magnetic field, $\bar{H}_{\text {inc }}$, is applied to the outside of the waveguide where an eddy current, $\bar{J}_{e}$, is formed on the outside surface by Faraday's law. When a slot is present the eddy current produces two fields: (i) a field anti-parallel to $\bar{H}_{\text {inc, }}$ reducing the surface current on the face of the waveguide everywhere but at the edges, and (ii) a field parallel to $\bar{H}_{\text {ine }}$ along the geometry of the slot. An electric field across the gap in the $\hat{\mathbf{z}}$ direction is produced by this current potential.

The slot thickness, $d$, and slot depth, $\rho$, are the two variables used to create the magnitude and shape of the electric field in the gap, primarily $E \hat{\mathbf{Z}}$. Boundary conditions require that $E_{\tan } \hat{\mathbf{z}}=0$ on the surface. This requirement produces a half-sinusoidal shape around a 2.5-dimensional ${ }^{1}$ slot length, with zero on the depth face. The electric field magnitude and direction are characterized using Ansys HFSS simulations. The field profile and amplitude is used in the calculation of the magnetic current source.

Since the wavelength of this problem is much greater than the size of the slot and the material is PEC, it is assumed that there is no

Journal of Magnetic Resonance, Vol 274 (January 2017): pg. 115-124. DOI. This article is @ Elsevier and permission has been granted for this version to appear in e-Publications@Marquette. Elsevier does not grant permission for this article to be further copied/distributed or hosted elsewhere without the express permission from Elsevier. 
attenuation or phase differences in the transmission of the electric field from the outside to the inside of the waveguide. This assumption allows for the exchange of the electric field in the slot to a magnetic current on the inside surface of the waveguide, defined by

\section{$\bar{M}_{s}=-\hat{\mathbf{n}} \times E \hat{\mathbf{z}}$,}

equation(41)

where $\hat{\mathbf{n}}$ is a normal vector facing into the waveguide on the interior surface. Finally, the equivalent source can be found by using the equivalence principle and image theory.

Characterization of the surface magnetic current source is illustrated in Fig. 4. Using a unit-step function the width of the cut slot as predicted by Born's first approximation [16], shown as a light dashed line, was found to be inadequate due to a secondary wave formed around the slot. It was found that creating a unit-step function 2.5 times larger than the slot width, shown as a light solid line, was needed to minimize the error on axis. However, a surface magnetic current in the shape of a unit function does not make physical sense. Therefore, in the formulation of the source, a Gaussian shaped surface magnetic current, $\bar{M}_{s}$, was characterized, illustrated in Fig. 4 as dashed black lines.

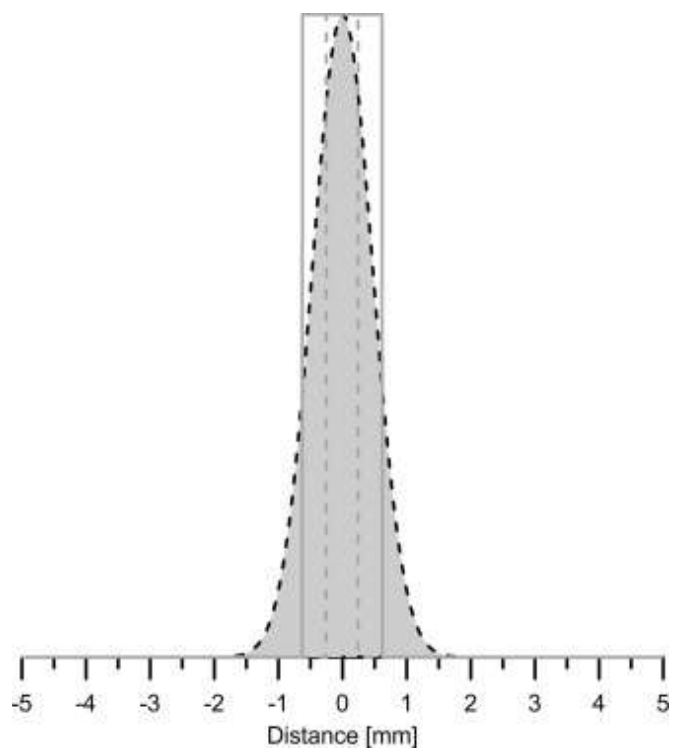

Fig. 4. Characterization of magnetic surface current source. A unit-step function is used as the surface magnetic current which fills the exact slot thickness (light dashed) 
as predicted by Born's first approximation. To minimize error, the unit-step function was made wider than the actual slot (light solid). However, surface magnetic current actually has a Gaussian shape (black dashed) that extends past the slot thickness.

Using a Gaussian shape gives physical insight to the distribution of the electric field and, therefore, the surface magnetic current source from the slot. Since the distribution of the surface magnetic current source is more complicated than a simple unit-step function, the equivalent surface magnetic current is replaced with a single magnetic current filament source, $\bar{M}_{f}$, located on the surface of the waveguide walls along the center of the slot. The solution to the problem is then solved by performing a posterior product of the surface magnetic current filament and Eq. (26) for a rectangular waveguide, and, likewise, using Eq. (40) for a cylindrical waveguide. To account for the distribution of magnetic current, the convolution of the solution with a properly characterized Gaussian function, $\mathbf{M}_{\text {conv, }}$ such as one described in Fig. 4, is used to describe the primary and secondary waves associated with the slot. Using the convolution, a complete solution can be formed.

\section{Results}

\subsection{Single-slot rectangular results}

The rectangular geometry used for the formulation of the solution is illustrated in Fig. 2. The lowest order mode in the rectangular waveguide with the dimensions $2.54 \times \times 1.27 \mathrm{~mm}$ is the $\mathrm{TE}_{10}$ with a cut-off frequency of $59.055 \mathrm{GHz}$, which makes $100 \mathrm{kHz}$ well below propagation cut-off. The propagation of all modes coupling into the waveguide are then evanescent.

A two-dimensional contour plot of the evanescent mode solutions using the dyadic Green's functions and the convolution techniques are shown in Fig. 5. The contour plots are of the normalized magnetic field magnitude ( $\mathrm{H}_{a b s}$ shown in Fig. $\left.5 \mathrm{~A}\right)$ and the individual vector components: $\mathrm{H}_{x}, \mathrm{H}_{y}$, and $\mathrm{H}_{z}$; Figs. $5 \mathrm{~B}, \mathrm{C}$, and $\mathrm{D}$, respectively. The slot source was a single slot cut into the right face of the waveguide in the $-\hat{y}$-direction with a slot thickness, $d$, of $0.05 \mathrm{~mm}$. For the initial results the slot depth was half the waveguide thickness; $\rho$ is $0.625 \mathrm{~mm}$. The slot cut is indicated in all figures as a

Journal of Magnetic Resonance, Vol 274 (January 2017): pg. 115-124. DOI. This article is (C) Elsevier and permission has been granted for this version to appear in e-Publications@Marquette. Elsevier does not grant permission for this article to be further copied/distributed or hosted elsewhere without the express permission from Elsevier. 
black outline. The applied magnetic field is uniform and in the $\hat{x}$ direction.
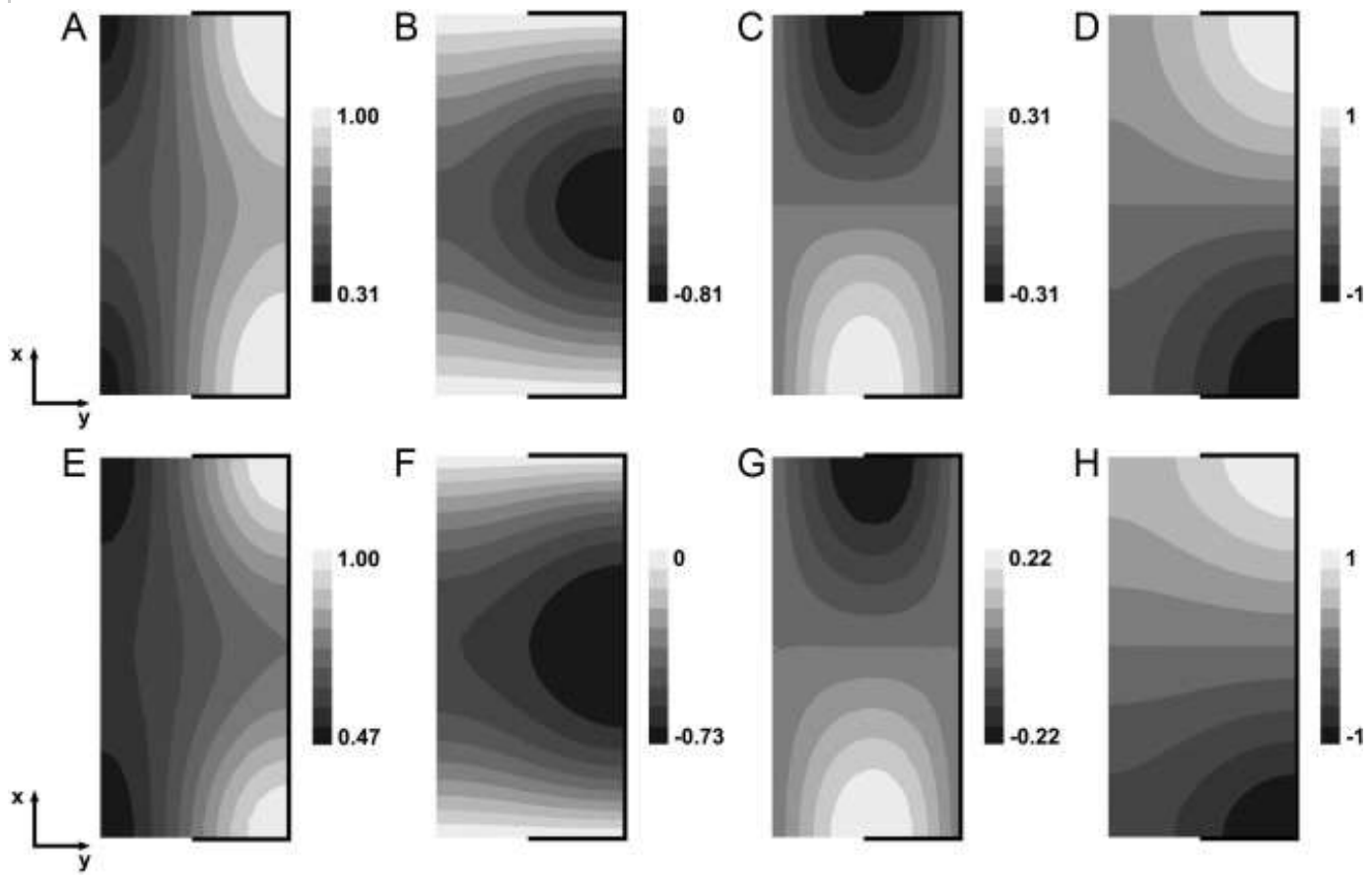

Fig. 5. Rectangular waveguide solutions using dyadic Green's functions. Slot cut $50 \%$ deep from right side. The magnetic field profile components are (A) magnetic field magnitude, (B) $\hat{x}$-component, $\mathrm{H}_{\mathrm{x}}$ (C) $\hat{y}$-component, $\mathrm{H}_{\mathrm{y}},(\mathrm{D}) \hat{z}$-component, $\mathrm{H}_{\mathrm{z}}$. Rectangular waveguide solutions using Ansys HFSS. The magnetic field profile components are (E) magnetic field magnitude, (F) $\hat{x}$-component, $\mathrm{H}_{\mathrm{x}},(\mathrm{G}) \hat{\hat{y}_{-}}$ component, $\mathrm{H}_{\mathrm{y}},(\mathrm{H}) \hat{\mathrm{Z}}$-component, $\mathrm{H}_{\mathrm{z}}$. Each contour represents a $10 \%$ change in amplitude.

The results are visually compared to Ansys HFSS data, shown in Fig. 5E-H. Good agreement between Green's function and numerical results is shown. The results from the dyadic Green's functions show a slightly larger gradient of magnetic field in the $\hat{y}$-direction.

\subsection{Solution along the $\pm \hat{z}$-axis}

Confidence in the magnetic field profile along the axis of the sample is crucial to the analysis of single- and multiple-slot configurations. Comparison of Ansys and dyadic Green's function solutions down the $\pm \hat{z}$-axis result in a RMSE of 0.307 , using Eq. (1). The results are plotted in Fig. 6 where the dyadic Green's function 
solution using the magnetic surface current filament and convolution technique is plotted alongside Ansys HFSS solutions. The upward trend of the magnetic field at the edges of the solution on axis is an artifact of the convolution. The last $0.5 \mathrm{~mm}$ on each end are ignored in the RMSE calculation. Without the artifacts the solution should decay at an exponential rate and this rate is used as the criteria for the comparison.

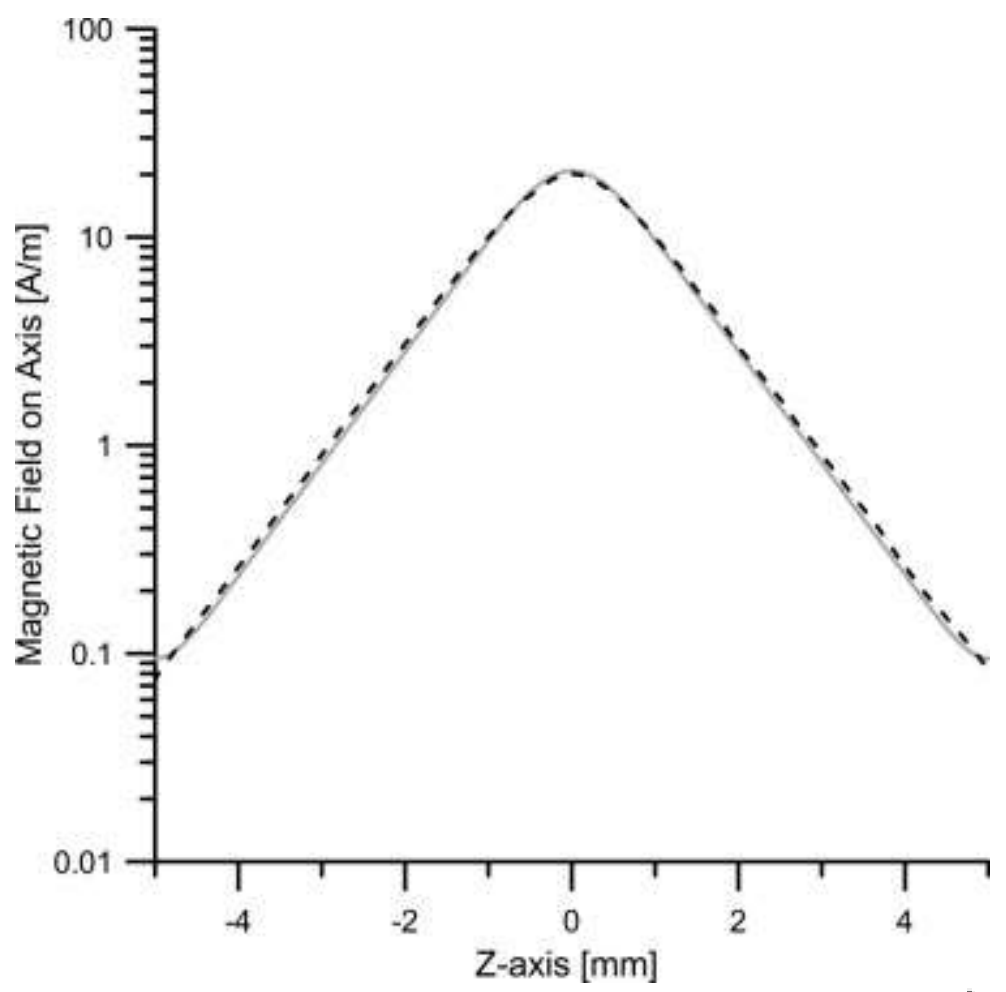

Fig. 6. Magnetic field profile for the rectangular waveguide $\hat{Z}$-axis. Plotted is the Ansys HFSS solutions (dashed) and the dyadic Green's function solution using the magnetic surface current filament and convolution technique, $M_{s}$ (solid). An RMSE of 0.307 was calculated.

\subsection{Single-slot cylindrical results}

The cylindrical geometry used for the formulation of the solution is illustrated in Fig. 3. The radius $a$ is $3.175 \mathrm{~mm}$ and the center of the slot is located on the $x y$-plane with a depth, $\rho$, of $3.175 \mathrm{~mm}$ and a thickness, $d$, of $0.05 \mathrm{~mm}$. The lowest order mode that propagates in the cylindrical waveguide with a radius of $3.175 \mathrm{~mm}$ is the $\mathrm{TE}_{11}$ with a cut-off frequency of $55.338 \mathrm{GHz}$, which makes $100 \mathrm{kHz}$ well below 
propagation cut-off. The propagation of all modes coupling into the waveguide are then evanescent.

Similar to the rectangular results, a sinusoidal electric field in the $\hat{\mathbf{Z}}$-direction is used to create the magnetic current around the slot.

A two-dimensional contour plot of the evanescent mode solutions using the dyadic Green's functions and the convolution techniques described previously, are shown in Fig. 7. The contour plots are of the normalized magnetic field magnitude ( $\mathrm{H}_{a b s}$ shown in Fig. 7A) and the individual vector components: $\mathrm{H}_{\mathrm{x}} \mathrm{H}_{\mathrm{y}}$, and $\mathrm{H}_{\mathrm{z}}$; Figs. 7B, $\mathrm{C}, \mathrm{D}$, respectively. The slot source was a single slot cut into the right face of the waveguide in the $-\hat{y}$-direction with a slot thickness, $d$, of $0.05 \mathrm{~mm}$. For the initial results the slot depth was half the waveguide thickness, $\rho$ is $3.175 \mathrm{~mm}$. The slot cut is indicated in all figures as a black outline. The applied magnetic field is uniform and in the $\hat{x}$ direction.
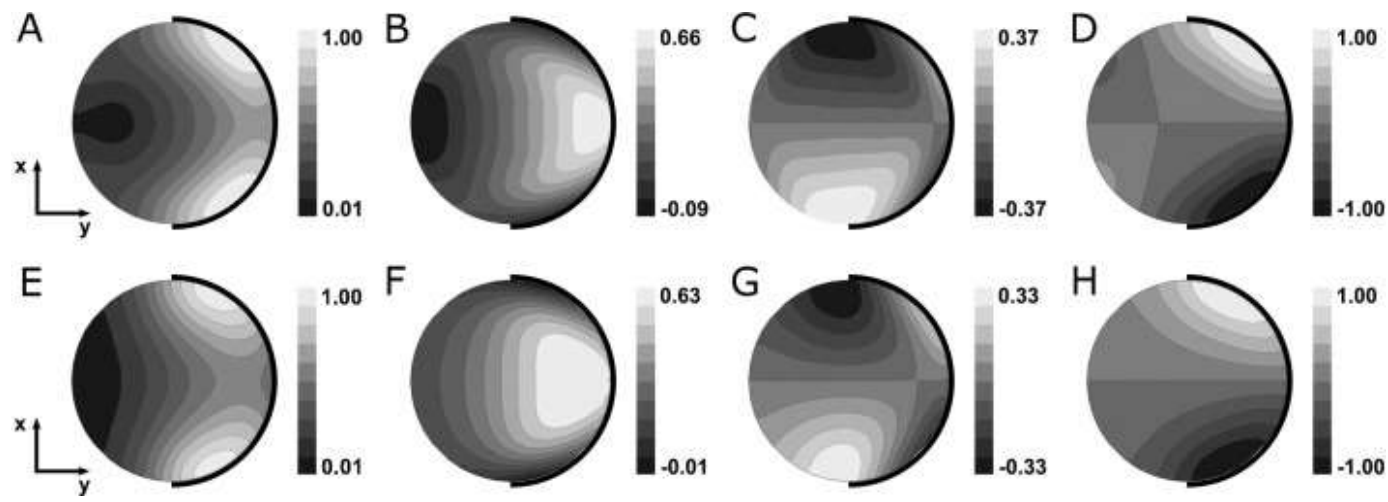

Fig. 7. Cylindrical waveguide solutions using dyadic Green's functions. Slot cut 50\% deep from right side. The magnetic field profile components are the (A) magnetic field magnitude, (B) $\hat{x}$-component, $\mathrm{H}_{\mathrm{x}}$ (C) $\hat{y}$-component, $\mathrm{H}_{\mathrm{y}},(D) \hat{z}$-component, $\mathrm{H}_{\mathrm{z}}$. Cylindrical waveguide solutions using Ansys HFSS. The magnetic field profile components are (E) magnetic field magnitude, $(\mathrm{F}) \hat{x}$-component, $\mathrm{H}_{\mathrm{x}},(\mathrm{G}) \hat{\mathrm{y}}$ component, $\mathrm{H}_{\mathrm{y}},(\mathrm{H}) \hat{\mathrm{Z}}$-component, $\mathrm{H}_{\mathrm{z}}$. Each contour represents a $10 \%$ change in amplitude.

The results are visually compared to Ansys HFSS data, shown in Fig. 7E-H. Good agreement between Green's function and numerical results are shown. The results from the dyadic Green's functions show a slightly larger gradient of magnetic field in the $\hat{y}_{\text {-direction. }}$

Journal of Magnetic Resonance, Vol 274 (January 2017): pg. 115-124. DOI. This article is (C) Elsevier and permission has been granted for this version to appear in e-Publications@Marquette. Elsevier does not grant permission for this article to be further copied/distributed or hosted elsewhere without the express permission from Elsevier. 
NOT THE PUBLISHED VERSION; this is the author's final, peer-reviewed manuscript. The published version may be accessed by following the link in the citation at the bottom of the page.

\subsection{Solution along the $\pm \hat{z}$-axis.}

Comparison of Ansys and dyadic Green's function solutions down the $\pm \hat{z}$-axis result in a RMSE of 0.221, using Eq. (1). The results are plotted in Fig. 8, where the dyadic Green's function solution using the magnetic surface current filament and convolution technique is plotted along side the Ansys HFSS solutions. Again, the upward trend of the magnetic field at the edges of the solution on axis is a byproduct of the convolution. The ends are ignored in the RMSE calculation.

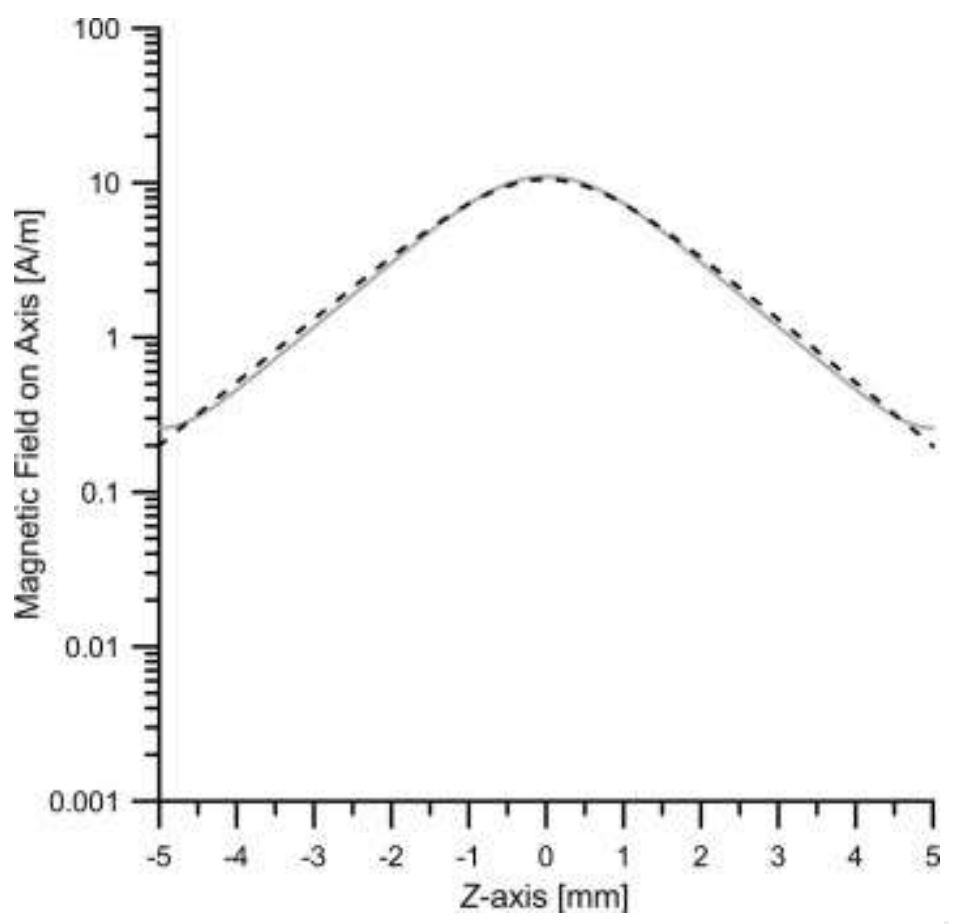

Fig. 8. Magnetic field profile for the cylindrical waveguide $\hat{Z}$-axis. Plotted is the Ansys HFSS solutions (dashed) and the dyadic Green's function solution using the magnetic surface current filament and convolution technique, $\mathrm{M}_{\mathrm{s}}$ (solid). An RMSE of 0.221 was calculated.

\section{Discussion}

\subsection{Multiple-slot formulation}

Solutions between Ansys HFSS single-slot simulations and dyadic Green's function solutions show good agreement for both cylindrical and rectangular results. Confidence in the magnetic field 
cross-section and amplitude along the axis is presented in the previous sections. From these single-slot solutions a multiple-slot formulation can be devised. Two methods are used in the formulation of the interactions between multiple slots: simple summation (superposition) of the slot fields and a first-order moment method to include mutual coupling of evanescent fields. The multiple-slot calculations were performed with the rectangular waveguide geometry but are also valid for cylindrical results.

\subsection{Summation of multiple-slot fields}

In the formulation of multiple slots, the dyadic Green's function were modified to displace the feed point in the $\tilde{z}$-direction. This is done by changing the $z$ ' variable in the dyadic Green's function in Eq. (26) or Eq. (40). Two solutions are then created at a $\pm z^{\prime}$ distance resulting in slot spacings of $5.08 \mathrm{~mm}, 2.54 \mathrm{~mm}$, and $0.508 \mathrm{~mm}$. The second slot depth, $\rho_{2}$, is then varied as $0.3 t, 0.5 t$, and $0.7 t$. These depths were chosen because they are practical depths in construction of field modulation slots. The solutions were simply summed together to create the initial zero-order results.

In the Ansys HFSS simulation, both slots were excited by a uniform magnetic field from outside the domain and the slots were varied. The results were then compared visually and the RMSE value was calculated using Eq. (1).

If the two slots act independently, a simple summation of the slot fields should completely describe the multiple-slot system. However, the Ansys HFSS calculations do not agree with the zeroorder dyadic Green's solution. Table 1 illustrates a number of experimental comparisons between Ansys HFSS and dyadic Green's function solution.

Table 1. RMSE values for simple summation formulation, $\mathrm{RMSE}_{s}$, and moment method, $\mathrm{RMSE}_{m}$, are directly compared for multiple slots of different depths.

\begin{tabular}{|c|c|c|c|c|}
\hline Distance $[\mathrm{mm}]$ & $\rho_{1} / t \rho 1 / t$ & $\rho_{2} / t \rho 2 / t$ & $\mathbf{R M S E}_{s}$ & $\mathrm{RMSE}_{m}$ \\
\hline 5.08 & 0.5 & 0.3 & 0.626165 & 0.508758 \\
\hline 5.08 & 0.5 & 0.5 & 0.501948 & 0.404064 \\
\hline 5.08 & 0.5 & 0.7 & 0.543898 & 0.781346 \\
\hline
\end{tabular}

Journal of Magnetic Resonance, Vol 274 (January 2017): pg. 115-124. DOI. This article is @ Elsevier and permission has been granted for this version to appear in e-Publications@Marquette. Elsevier does not grant permission for this article to be further copied/distributed or hosted elsewhere without the express permission from Elsevier. 
NOT THE PUBLISHED VERSION; this is the author's final, peer-reviewed manuscript. The published version may be accessed by following the link in the citation at the bottom of the page.

\begin{tabular}{|c|c|c|c|c|}
\hline Distance $[\mathrm{mm}]$ & $\rho_{1} / t \rho 1 / t$ & $\rho_{2} / t \rho 2 / t$ & $\mathrm{RMSE}_{s}$ & $\mathbf{R M S E}_{m}$ \\
\hline 2.54 & 0.5 & 0.3 & 0.683480 & 0.489288 \\
\hline 2.54 & 0.5 & 0.5 & 0.639576 & 0.427618 \\
\hline 2.54 & 0.5 & 0.7 & 0.535444 & 1.026530 \\
\hline 0.508 & 0.5 & 0.3 & 1.181602 & 0.415276 \\
\hline 0.508 & 0.5 & 0.5 & 1.372851 & 1.153870 \\
\hline 0.508 & 0.5 & 0.7 & 1.331543 & 2.894110 \\
\hline 0.254 & 0.5 & 0.3 & 2.29347 & 0.478667 \\
\hline 0.254 & 0.5 & 0.5 & 2.30797 & 1.087350 \\
\hline 0.254 & 0.5 & 0.7 & 2.24897 & 3.034104 \\
\hline
\end{tabular}

When the solutions are "far enough" apart, ${ }^{2}$ they have little interaction. This is shown in Table 1 under $\mathrm{RMSE}_{s}$, where the slots are $5.08 \mathrm{~mm}$ apart and the RMSE for the slots with the same depth are comparable to the single-slot RMSE of 0.307 . The majority of the errors arise from the region between the slots. It appears that the effective roll-off between the slots is shifted. Increasing the number of modes in the dyadic Green's function solution had no effect in reducing the error between the slots.

As the slots are moved closer together to $2.54 \mathrm{~mm}$ apart the interactions of the slots start to change. In general, the RMSE rises only slightly but the region containing error is significantly different. More error arises from the region at the slots, which has the largest effect on the RMSE and the validity of the solution. Finally, when the slots become close the amplitude of the Ansys HFSS solutions are significantly smaller than the simple summation of the slot fields from the dyadic Green's functions.

The summation of the slots only takes into account the magnetic field from the excitation of the slots. Since the physical slots were removed in the formulation of the dyadic Green's functions, the mutual coupling that may occur between the slots is neglected. Mutual coupling is defined as the interactions between the two slots that are not accounted for by the excitation of the individual slots.

The mutual coupling between multiple slots must be accounted for in order to reduce the error between the Ansys HFSS and dyadic Green's function solutions. In this work, a first-order moment method

Journal of Magnetic Resonance, Vol 274 (January 2017): pg. 115-124. DOI. This article is @ Elsevier and permission has been granted for this version to appear in e-Publications@Marquette. Elsevier does not grant permission for this article to be further copied/distributed or hosted elsewhere without the express permission from Elsevier. 
is implemented in order to characterize and fully account for the phenomenon of mutual coupling.

\subsection{Moment method}

The moment method is a way to describe the total response (caused by two or more stimuli) at a given point as the sum of the individual responses and the sum of the responses on each stimuli caused by the other stimuli. In this work the magnetic field is found to be 180 degrees out of phase from the primary magnetic field of the second slot, reducing the overall effect of the slot solution. At the same time the reciprocal problem is occurring.

The moment method used is said to be of "first-order". In this work, first-order is described as the summation of the independent slots (zero-order) and the mutual-coupling interaction between slots (first-order). Standard S-parameter notation will be used to describe the mutual coupling where the second index is reserved for the excitation and the first for the position. Thus $\bar{H}_{12}$ describes the magnetic field at slot one, excited by slot two.

The moment method has been used in the literature as an evaluation technique to calculate the mutual coupling between microstrip antenna arrays [17]; [18]; [19] ; [20]. Using reciprocity, a voltage is applied on one slot and a current is measured across the other slot. This work follows the methods described by Pan and Pozar, [17] ; [18] respectively.

In Refs. [17] ; [18] a voltage reflection current is defined on the surface from an incident field driven by the ith slot onto the $j$ th slot. The reflection magnetic current is defined as

$$
\bar{R}_{j i}=i \omega \mu \int \overline{\bar{G}}_{m}^{j} \cdot \bar{M}_{f}^{i j} d \Omega,
$$

equation(42)

where $M_{f}^{i j}$ is the $j$ th filament magnetic current centered at the position of the $j$ th slot calculated from the $i$ th source, $\overline{\bar{G}}_{m}^{j}$ is the expected modal solution of the magnetic field from the dyadic Green's functions located at the $j$ th slot and $\Omega$ is the 2.5-dimensional line around the 
waveguide walls centered at the $j$ th slot. From here the magnetic field driven from the ith slot at the $j$ th slot is calculated by

$$
\bar{H}_{j i}=\int \overline{\bar{G}}_{m}^{j} \cdot \bar{R}_{j i} d \Omega \text { for } i \neq j \text {. }
$$

Since this work uses only two slots, the magnetic current can be solved directly using the dyadic Green's functions. Knowing the magnetic field from the first slot, the magnetic current on the second slot can be calculated. If more slots were being considered a matrix would be created taking into effect all slot interactions. The total solution is then the summation of the slots plus the first-order solution, such that

$\bar{H}_{\text {tot }}=\bar{H}_{11}+\bar{H}_{22}+\left(\bar{H}_{21}+\bar{H}_{12}\right)$.

It should be noted that $\bar{H}_{21}$ and $\bar{H}_{12}$ result in magnetic fields that oppose the superimposed fields, yielding a lower total magnetic field as expected.

Using the moment method, solutions were calculated for slot distances of $5.08 \mathrm{~mm}, 2.54 \mathrm{~mm}$ and $0.508 \mathrm{~mm}$. In these calculations the first slot depth, $\rho_{1} \rho 1$, constant at $0.5 t$ while the second slot depth, $\rho_{2}$, is varied between $0.3 t, 0.5 t$, and $0.7 t$. The results of these calculations can be found in Table 1 under $\mathrm{RMSE}_{m}$.

Table 1 shows some mixed results between the RMSE of the simple summation solutions, $\mathrm{RMSE}_{s}$, and the first-order method moment, $\mathrm{RMSE}_{m}$. For variations of slot distance and slot depths of $0.3 t$ and $0.5 t$, the moment method decreased the RMSE as expected. The issue arises in the results where the second slot depth was $0.7 t$, where in all cases the RMSE increased.

The issue of the increased RMSE in the case where the second slot depth is $0.7 t$ is not due to the accuracy of the moment method instead, it is due to changes in the system from outside of the domain. To test this hypothesis the two slots were moved to $0.254 \mathrm{~mm}$ apart which would increase mutual coupling. If the anomaly is from the 
moment method, then increasing the mutual coupling should increase the error. This is shown not to be the case by observing Table 1 . Here the exact phenomenon of an increasing RMSE occurs at the second slot depth of $0.7 t$ at a distance of $0.254 \mathrm{~mm}$ while the other two slot depths are improved.

Using Ansys simulations, a strong mutual coupling between the two slots and a relatively unperturbed surface current located on the opposite side of the slot is observed for the $0.3 t$ and $0.5 t$ cases. However, in the case of $0.7 t$ a different phenomenon, not included within the domain, is seen in the interaction of the currents on the face of the waveguide. From this interaction the mutual coupling decreases resulting in an inaccurate moment method interaction.

If each slot were driven from independent sources, the addition of the slot magnetic fields and their first-order interactions would be sufficient to obtain the field profile down axis. Instead, all slots are driven with a single incident wave and the electric field in the slot is dependent on eddy currents formed by scattered waves outside the domain. The results are non-trivial under some conditions. With this complexity arising from slot-to-slot interaction outside the domain, it would prove difficult to fully express in an Green's function solution for slot depths deeper than $0.6 t$.

\subsection{Field modulation cross-section incident on sample}

In EPR, only the components of $\bar{H}$ parallel to the static magnetic field create the field modulation effect. In the presented geometry it is the $\hat{x}$-component. Due to the standing-wave modes formed by the slot excitation, the $\mathrm{H}_{\mathrm{x}}$ component of the magnetic field does not have a uniform cross-section, shown in Fig. 9. 

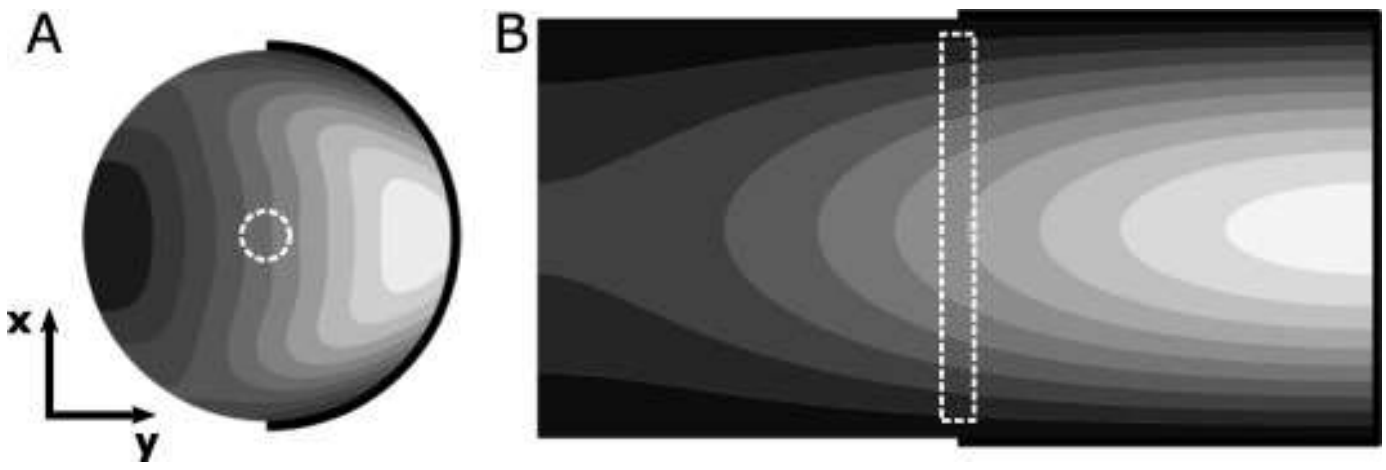

Fig. 9. $E P R(A)$ cylindrical $T E_{011}$ cavity and $(B)$ rectangular $T E_{102}$ cavity showing the $100 \mathrm{kHz} \hat{X}$-component, $\mathrm{H}_{\mathrm{x}}$, solutions using dyadic Green's functions. The solid line illustrates the slot and the dashed line illustrates a capillary or flat-cell sample placed in the cavity, respectively.

The cross-sectional field modulation profile for a cylindrical $\mathrm{TE}_{011}$ cavity is shown in Fig. 9A. Here a capillary outline is placed in the center of the cavity and shows a $10 \%$ gradient of magnetic field through the sample for a slot with a depth of $0.5 t$. Further studies involving variation of the rotation of the slot cut may yield a more uniform cross-section.

In a rectangular $\mathrm{TE}_{102}$ cavity only one slot can be cut in the center of the cavity in the $x$-plane. Otherwise microwave leakage will occur. The cut in the $y$-plane, as shown in Fig. 2, does not allow if propagation without rf leakage. However, the use of a uniform rf field rectangular cavity TEU02 allows for multiple slots to be cut in the $x$ plane without degradation of the mode [9]; [10] ; [11]. Solutions for the $\hat{x}$-component with a cut in the $x$-plane are straightforward and shown in Fig. 9B.

If a flat-cell sample of some arbitrary thickness, shown as a dashed line, were to be placed down the axis of the rectangular microwave cavity, it is clear that the differential field modulation amplitude would vary in both $\hat{x}$ and $\hat{y}$ directions, along with the evanescent roll off in $\pm \hat{z}$-directions. This is not apparent in current EPR literature, where the quasi-static field is assumed to create modes that are uniform over the cross-section of the sample.

\subsection{W-band cylindrical TE $01 \cup$ cavity with uniform $100 \mathrm{kHz}$ field modulation profile}

Journal of Magnetic Resonance, Vol 274 (January 2017): pg. 115-124. DOI. This article is @ Elsevier and permission has been granted for this version to appear in e-Publications@Marquette. Elsevier does not grant permission for this article to be further copied/distributed or hosted elsewhere without the express permission from Elsevier. 
The uniformity of the rf magnetic field in a microwave cavity, where typically a cavity with modulation slots has a cosine dependence in the propagation direction, has been discussed in great detail [9]; [10] ; [11]. The geometry of a resonator designed for W-band cylindrical $\mathrm{TE}_{01 \mathrm{u}}$ cavity with over-sized end sections is described in Table 2 and a cross section is shown in Fig. 10A. This design uses two metallic screws as sample shields placed in the end-sections to more abruptly stop the incident microwave magnetic field. These screws also allow for frequency adjustment to match the cutoff section. Microwave magnetic field uniformity is $85 \%$ in $6 \mathrm{~mm}$ region of interest.

Table 2. Design parameters for the $\mathrm{W}$-band cylindrical $\mathrm{TE}_{01 \mathrm{U}}$ with uniform $100 \mathrm{kHz}$ field modulation.

\section{Feature description}

Outer diameter, $a$

Inner diameter

End section height

End section diameter

Modulation slot thickness

Modulation slot depth, $\rho$

(symmetric about center)

\section{Dimension}

$6.35 \mathrm{~mm}$

$3.96 \mathrm{~mm}$

$1.72 \mathrm{~mm}$

$5.59 \mathrm{~mm}$

$0.05 \mathrm{~mm}$

$0.56 t, 0.56 t, 0.64 t, 0.48 t$

$0.56 t, 0.76 t, 0.56 t$
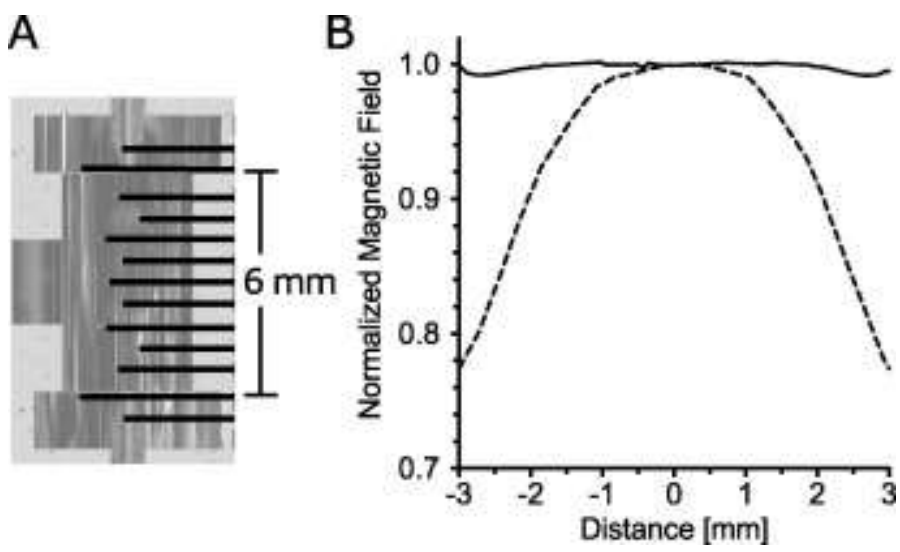

Fig. 10. (A) Geometry of the W-band cylindrical $T E_{010}$ with uniform rf field and designed slots to produce uniform $100 \mathrm{kHz}$ incident along the axis of the sample. Slots are shown in black along the $6 \mathrm{~mm}$ region of interest. Using Ansys HFSS, (B) Normalized $100 \mathrm{kHz} \hat{X}$-component, $\mathrm{H}_{\mathrm{x}}$, magnetic field plot of the optimized slot geometry (solid) and $0.75 \rho$ uniform slot depth (dashed).

Field modulation slots are cut transverse to the magnetic field in a pattern optimized to exhibit a uniform $100 \mathrm{kHz}$ field modulation along the axis of the sample. This pattern was solved by hand with 
insights gained from the formation of the dyadic Green's functions. The design methodology is to start with a $0.5 t$ uniform slot depth and adjust the top two (symmetric with bottom) slots till the field exceeds the center. Then the central slots are adjusted to accommodate. This is not a unique solution. Future work could automate this process using the first-order methods of moments dyadic Green's functions as an initial optimization.

Ansys HFSS simulations of this geometry are shown in Fig. 10B (solid) and compared to the field modulation profile of slots with a uniform depth of $0.7 t$ (dashed). The field modulation excitation of the optimized slot geometry is designed with less than $1 \%$ fluctuations over the $6 \mathrm{~mm}$ region of interest.

\section{Conclusion}

Dyadic Green's functions have been useful in understanding evanescent field coupling to slots cut into waveguide walls. By using a Gaussian function instead of a unit-step function, as assumed by Born's first approximation, near-field secondary wave effects are included to produce an accurate profile of the magnetic current inside a waveguide for slots much smaller than a wavelength.

Interactions arising from multiple slots can be understood using a combination of simple summation and first-order mutual coupling techniques to account for the slot interaction. However, the Green's function methods are limited due to eddy current changes outside the domain that cannot be accounted for directly in the dyadic Green's function solution for some conditions. For final evaluation, Ansys HFSS solutions have proven to be accurate by simulating the full geometry.

Electromagnetic modes forming from the $100 \mathrm{kHz}$ time-varying source are shown to have a complex cross-section at the sample which has not been previously documented in EPR literature. With an electromagnetic cross-section that is not uniform, the placement of the sample and overall sample geometry will have an effect on the magnetic field modulation applied to the sample. Additionally, this work shows the feasibility of using different slot depths or slot angles to change the interior magnetic field profile to be more favorable for

Journal of Magnetic Resonance, Vol 274 (January 2017): pg. 115-124. DOI. This article is (C) Elsevier and permission has been granted for this version to appear in e-Publications@Marquette. Elsevier does not grant permission for this article to be further copied/distributed or hosted elsewhere without the express permission from Elsevier. 
NOT THE PUBLISHED VERSION; this is the author's final, peer-reviewed manuscript. The published version may be accessed by following the link in the citation at the bottom of the page.

EPR spectroscopy. A design of a cylindrical TE $\mathrm{T}_{01 \mathrm{U}}$ cavity is presented with both uniform rf field and $100 \mathrm{kHz}$ modulation field.

\section{Acknowledgements}

This work was supported by Grants EB001417 and EB001980, National Institute of Biomedical Imaging and Bioengineering (NIBIB), National Institutes of Health (NIH).

\section{References}

[1] C.P. Poole. Electron Spin Resonance: A Comprehensive Treatise on Experimental Techniques. Interscience (1967)

[2] R.R. Mett, J.R. Anderson, J.W. Sidabras, J.S. Hyde. Electron paramagnetic resonance field-modulation eddy-current analysis of silver-plated graphite resonators. Rev. Sci. Instrum., 76 (9) (2005), p. 094702

[3] O.Y. Grinberg, L.J. Berliner (Eds.), Very High Frequency (VHF) ESR/EPR, Springer Press (2004)

[4] K. Möbius, A. Savitsky. High-field EPR Spectroscopy on Proteins and Their Model Systems: Characterization of Transient Paramagnetic States. Royal Society of Chemistry (2009) https://books.google.de/books?id=fbQtqM1nxDMC

[5] O. Burghaus, M. Rohrer, T. Gotzinger, M. Plato, K. Mobius. A novel highfield/high-frequency epr and endor spectrometer operating at $3 \mathrm{~mm}$ wavelength. Meas. Sci. Tech., 3 (8) (1992), p. 765

[6] G.R. Eaton, S.S. Eaton, D.P. Barr, R.T. Weber. Quantitative EPR. Springer, Wien, New York (1941)

[7] C. Tai. Dyadic Green's Functions in Electromagnetic Theory. (first ed.) International Textbook (1971)

[8] C. Tai. Dyadic Green's Functions in Electromagnetic Theory. (second ed.)IEEE Press (1994)

[9] R.R. Mett, W. Froncisz, J.S. Hyde. Axially uniform resonant cavity modes for potential use in electron paramagnetic resonance spectroscopy. Rev. Sci. Instrum., 72 (2001), p. 4003

[10] J.R. Anderson, R.R. Mett, W. Froncisz, J.S. Hyde. Cavities with axially uniform fields for use in electron paramagnetic resonance. II. Free space generalization. Rev. Sci. Instrum., 73 (2002), p. 3027

[11] J.S. Hyde, J.R. Anderson, R.R. Mett. Cavities with axially uniform fields for use in electron paramagnetic resonance. III. Re-entrant geometries. Rev. Sci. Instrum., 73 (2002), p. 4003

[12] P.M. Morse, H. Feshback. Methods of Theoretical Physics: Part I. McGraw-Hill (1953)

Journal of Magnetic Resonance, Vol 274 (January 2017): pg. 115-124. DOI. This article is @ Elsevier and permission has been granted for this version to appear in e-Publications@Marquette. Elsevier does not grant permission for this article to be further copied/distributed or hosted elsewhere without the express permission from Elsevier. 
[13] P.M. Morse, H. Feshback. Methods of Theoretical Physics: Part II. McGraw-Hill (1953)

[14] J.B. Conway. Functions of One Complex Variable. (second ed.) SpringerVerlag, New York, NY (1978)

[15] L.W. Li, P.S. Kooi, M.S. Leong, T.S. Yeo, S.L. Ho. On the eigenfunction expansion of electromagnetic dyadic greens functions in rectangular cavities and waveguides. IEEE Trans. Microw. Theory Techn., 43 (3) (1995), pp. 700-702

[16] M. Born, W. Wolf. Principles of Optics: Electromagnetic Theory of Propagation, Interference and Diffraction of Light. (third ed.)Pergamon Press (1965)

[17] S. Pan. Computation of mutual coupling between slot-coupled microstrip patches in a finite array. IEEE Trans. Antennas. Propag., 40 (9) (1992), p. 1047

[18] D.M. Pozar. A reciprocity method of analysis for printed slot and slotcoupled microstrip antennas. IEEE Trans. Antennas. Propag., AP-32 (12) (1986), p. 1439

[19] P.B. Katehi. Mutual coupling between microstrip dipoles in multielement arrays. IEEE Trans. Antennas. Propag., 37 (3) (1989), p. 275

[20] D.M. Pozar. Input impedance and mutual coupling of rectangular microstrip antennas. IEEE Trans. Antennas. Propag., AP-30 (6) (1982), p. 1191

${ }^{1}$ The term 2.5-dimensional is defined as a two-dimensional area that spans multiple normals. In this case, the waveguide has two normal vectors in the $\pm \hat{x}$-direction and one in the $-\hat{y}$-direction. Resulting in three combined two-dimensional areas with multiple normal vectors.

2The term "far enough" is defined by observing the evanescent roll-off of each peak individually. In this work, if the evanescent field is more than a factor of ten down in amplitude by the time it reaches the other slot position it is defined as "far enough".

Journal of Magnetic Resonance, Vol 274 (January 2017): pg. 115-124. DOI. This article is @ Elsevier and permission has been granted for this version to appear in e-Publications@Marquette. Elsevier does not grant permission for this article to be further copied/distributed or hosted elsewhere without the express permission from Elsevier. 